\title{
The Microstructure of Currency Markets
}

\author{
Martin D. D. Evans* \\ Department of Economics \\ Georgetown University \\ and NBER
}

July 2010

\begin{abstract}
This article summarizes exchange-rate research using microstructure models. It first lays out the key features of the foreign exchange market and describes how they are incorporated into a canonical model of currency trading. The empirical implications of the model are then examined. The article also discusses how currency trading links spot rate dynamics to macroeconomic conditions, and how this link sheds light on some long-standing puzzles concerning the behavior of exchange rates.
\end{abstract}

\section{Key Words:}

Currency Trading, Exchange Rates, Exchange Rate Puzzles, Exchange Rate Fundamentals, Foreign Exchange Market, Microstructure, Order Flow, Risk Premium.

\section{Introduction}

Models of Foreign Exchange Market Microstructure examine the determination and behavior of spot exchange rates in an environment that replicates the key features of trading in the foreign exchange (FX) market. Traditional macro exchange rate models play little attention to how trading in the FX market actually takes place. The implicit assumption is that the details of trading (i.e., who quotes currency prices and how trade takes place) are unimportant for the behavior of exchange rates over months, quarters or longer. Micro-based models, by contrast, examine how information relevant to the pricing of foreign currency becomes reflected in the spot exchange rate via the trading process. According to this view, trading is not an ancillary market activity that can be ignored when considering exchange rate behavior. Rather, trading is an integral part of the process through which spot rates are determined and evolve.

The past decade has witnessed rapid growth in micro-based exchange-rate research. Originally the focus was on partial equilibrium models that captured the key features of FX trading. These models provided a new and rich array of empirical predictions that are strongly supported by the data and provide a new perspective on the proximate drivers of exchange rates over short horizons, ranging from a few minutes to

*The paper was prepared as an entry for the Encyclopedia of Financial Globalization. 
a few weeks. Recent micro-based research moves away from the traditional partial equilibrium domain of microstructure models to focus on the link between currency trading and macroeconomic conditions. This research aims to provide the micro-foundations for the exchange-rate dynamics that having been missing from general equilibrium macro models.

I structure my discussion of micro-based exchange-rate research as follows: Section 2 first lays out the key features of the FX market and describes how they are incorporated into a canonical model of currency trading. I then discuss the empirical implications of the model. Section 3 examines research that links spot rate dynamics to macroeconomic conditions via currency trading. Section 4 discusses how this research sheds light on some long-standing puzzles concerning the behavior of exchange rates. Section 5 concludes with some thoughts on the directions of future micro-based exchange-rate research.

\section{Currency Trading Models}

The goal of micro-based models is to build an exchange rate model from microeconomic foundations that reasonably represent the key features of the FX market. In particular, the aim is to incorporate the institutional implications of how information is transmitted from one agent to another as trading takes place, and to study how this information flow is ultimately reflected in the spot exchange rate. The fact that the models describe trading between agents does not mean that the researchers using these models are only interested in trading. Their focus remains on understanding exchange rate dynamics, but they are using models that make detailed predictions about trading activity as well.

\subsection{Features of the FX market}

No model can incorporate all the institutional features of trading in the FX market - its far too complex. Instead, micro-based models focus on a small number of features that are essential for understanding the main economic mechanisms at work. I summaries the key features below:

- The FX market is a two-tier market comprising the interbank and retail markets: Trading takes place between dealers working at banks in the interbank market, and between banks and their non-bank customers in the retail tier. Neither market has a physical location. Trading takes place by phone or electronically between participants located all around the world, but most trading in the interbank market takes place between banks located in a few financial centers: Tokyo, Singapore, Frankfurt, New York, and particularly London. Trading can take place 24 hours a day, but activity is heavily concentrated during the daytime hours of the main financial centers.

- FX dealers trade directly and indirectly in the interbank market. Direct interdealer trades result from "conversations" between two dealers working at different banks in which they agree to exchange one currency for another. This was the dominant form of interbank trading before the mid 1990s. Since then most interbank trade has taken the indirect form in which dealers submit market and limit orders to buy and sell currencies to electronic brokerages ( Reuters D2000-2 and EBS). These systems prioritize the limit orders so that those with the best prices are matched first with incoming market orders.

- No FX dealer has complete information about the state of the interbank market. Electronic brokerages provide market-wide information on transaction prices, but dealers do not observe the structure of limit 
orders that determine market liquidity. Direct interdealer trading takes place simultaneously across the interbank market. Dealers only have information on the trades they participate in.

- FX dealers face constraints on both the duration and size of their FX positions. Dealers' overnight positions are typically small or zero.

- Banks fill customer orders for currency in the retail tier of the market. The customer orders received by banks represent the most important source of private information to FX dealers. Dealers working at banks with a large customer base and a world-wide reporting system have a potentially important informational advantage over other market participants.

- Customer orders come from many different agent types and may be generated by allocative, speculative and risk-management factors. Customer orders that are purely a function of current and past currency prices are termed feedback orders.

\subsection{The Portfolio Shifts Model}

The Portfolio Shifts (PS) model incorporates many of these features into a model that examines how trading in the interbank and retail tiers of the FX market relates to the evolution of the spot exchange rate. The model describes trading in a single currency pair among a large number of dealers, a broker and between dealers and investors over a stylized trading day.

\subsubsection{Overview}

There is one risky asset representing FX and one risk-free asset with a daily return of $1+r$. The economy is populated by a continuum of risk-averse investors indexed by $n \in[0,1]$ and D risk-averse dealers indexed by $d$. Each day $t$, is split into three trading rounds; I, II and III. The sequence of events throughout day $t$ is shown in Figure 1.

At the start of round I on day $t$, all investors and dealers observe the dividend paid to the current holders of FX. The value of the dividend, $D_{t}$, is assumed to follow a random walk

$$
D_{t}=D_{t-1}+V_{t}
$$

where $V_{t} \sim$ i.i.d.N $\left(0, \sigma_{v}^{2}\right)$. Realizations of $V_{t}$ represent the arrival of public macroeconomic information over time (e.g., changes in interest rates). At the start of the day each investor $n$ also receives FX income,

$$
Y_{n, t}=Y_{t}+\xi_{n, t}
$$

where $Y_{t}$ and $\xi_{n, t}$ are i.i.d. mean zero normal random variables that represent the aggregate and idiosyncratic components of income. $Y_{n, t}$ is private information to each investor and generates a hedging motive for the customer orders in period I trading. Investors do not initially observe either component, but in equilibrium they learn the value of $Y_{t}$ by the end of day $t$.

The retail tier of the market opens in round I. Each dealer simultaneously and independently quotes a scalar price at which they will fill customer orders to buy or sell FX. The round-I price quoted by dealer $d$ is 


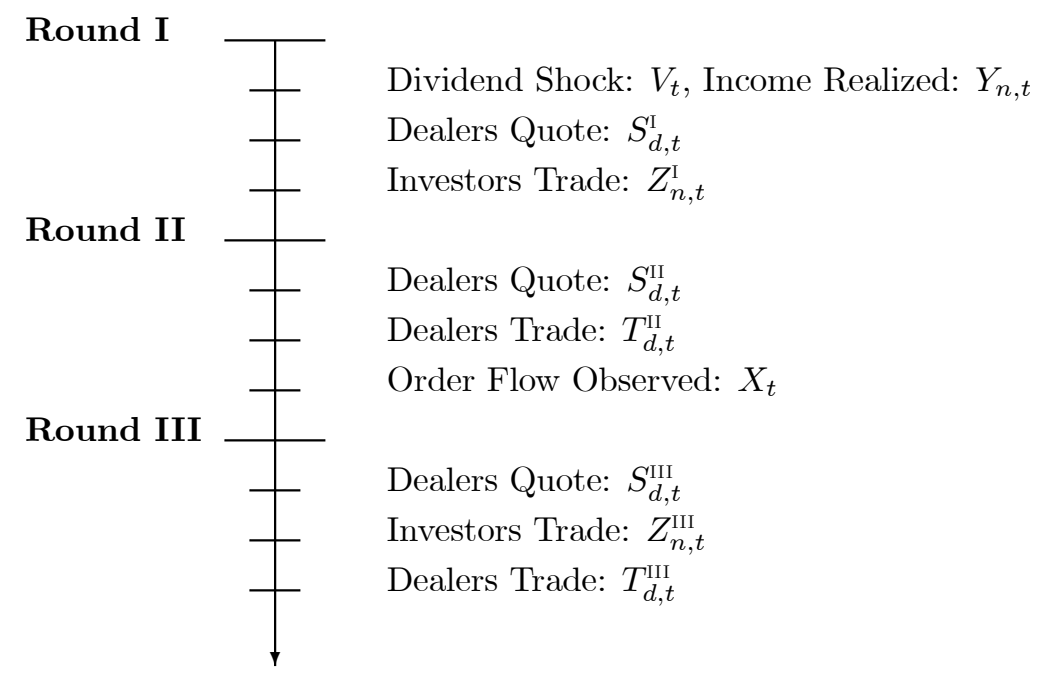

Figure 1: Daily Timing in the Portfolio Shifts Model

denoted by $S_{d, t}^{\mathrm{I}}$. Prices are publicly observed by all dealers and investors and are good for orders of any size. Investors then place their orders for FX. Orders may be placed with more than one dealer. If two or more dealers quote the same price, the customer order is randomly assigned among them. The customer orders received by dealer $d$ are denoted by $Z_{d, t}^{\mathrm{I}}$. Positive (negative) values of $Z_{d, t}^{\mathrm{I}}$ denote net customer purchases (sales) of FX. As in the actual retail market, customer orders, $Z_{d, t}^{\mathrm{I}}$, are only observed by dealer $d$.

Trading in the interbank tier of the market takes place in round II. The broker and each dealer simultaneously and independently quotes a scalar price for FX, $S_{\mathrm{B}, t}^{\mathrm{II}}$ and $\left\{S_{d, t}^{\mathrm{II}}\right\}_{d=1}^{\mathrm{D}}$. The quoted prices are observed by all dealers and are good for inter-dealer trades of any size. Each dealer then simultaneously and independently trades on other dealers' and the broker's quotes. I denote the FX orders made by dealer $d$ as $T_{d, t}^{\mathrm{II}}$ and orders received by dealer $d$ as $Z_{d, t}^{\mathrm{II}}$. Orders received by the broker are denoted by $Z_{\mathrm{B}, t}^{\mathrm{II}}$. When dealer $d$ initiates a purchases (sale) of FX, $T_{d, t}^{\mathrm{II}}$ is positive (negative). Positive values of $Z_{d, t}^{\mathrm{II}}$ or $Z_{\mathrm{B}, t}^{\mathrm{II}}$ denote purchases of FX initiated by another dealer. Once again, trading with multiple dealers and the broker is feasible. If multiple agents quote the same price, trades are allocated equally between them. At the close of round II trading, all dealers and the broker observe aggregate interdealer order flow:

$$
X_{t}=\sum_{d=1}^{\mathrm{D}} T_{d, t}^{\mathrm{II}}
$$

Interdealer order flow, $X_{t}$, simply aggregates the purchases of FX made by dealers initiating trades against other dealers' quotes and the broker's quote. Notice that order flow is different from trading volume. Obviously there is a buyer and seller of FX in every trade, but only one dealer initiates each trade. Order flow measures the direction of trading by identifying whether the buyer or seller of FX initiated the trade. As we will see, order flow plays a very important role in this model and in micro-based exchange-rate research more generally. 
The retail tier of the market re-opens in round III. The broker and dealers again simultaneously and independently quote prices, $S_{\mathrm{B}, t}^{\mathrm{III}}$ and $\left\{S_{d, t}^{\mathrm{III}}\right\}_{d=1}^{\mathrm{D}}$, at which they will fill dealer and customer orders, respectively. Investors observe all the prices and then place their orders with dealers. As above, orders may be placed with more than one dealer and are randomly assigned to dealers quoting the same prices. The round III customer orders received by dealer $d$ are denoted by $Z_{d, t}^{\mathrm{III}}$. Once each dealer has filled his customer orders, he can trade with the broker.

Clearly, this sequence of events represents a simplified view of trading in the actual foreign exchange market. It does, however, allow us to analyze how information pertinent to the spot exchange rate is learnt by dealers via trading. In particular, the focus of the model is on how information about the aggregate demand for FX contained in the customer orders at the start of the day becomes learnt by dealers and reflected in the FX prices they quote at the end of day. As we shall see, this information aggregation process produces an important empirical prediction linking trading activity to exchange rate dynamics.

The model's equilibrium comprises: (i) a set of optimal FX orders by investors in rounds I and III; (ii) a set of optimal FX price quotes by dealers in rounds I - III, and the broker in rounds II and III; and (iii) a set of optimal dealer trading decisions in rounds II and III, such that markets clear (i.e., there is a counterparty for every trade in each round).

\subsubsection{Equilibrium}

When all market participants act to maximize expected utility defined over future wealth, the equilibrium of the model is summarized by:

1. All dealers quote the same price for FX in each round of trading, i.e. $S_{d, t}^{i}=S_{t}^{i}$ for $i=\{\mathrm{I}, \mathrm{II}, \mathrm{III}\}$. The broker quotes the same price as dealers in rounds II and III. Common quotes are given by

$$
\begin{aligned}
S_{t}^{\mathrm{I}} & =S_{t-1}^{\mathrm{III}}-\lambda_{\mathrm{A}} A_{t-1}+\frac{1}{r} V_{t}, \\
S_{t}^{\mathrm{II}} & =S_{t}^{\mathrm{I}}, \quad \text { and } \\
S_{t}^{\mathrm{III}} & =S_{t}^{\mathrm{II}}+\lambda_{\mathrm{A}} A_{t-1}+\lambda_{\mathrm{X}}\left(X_{t}-\mathbb{E}\left[X_{t} \mid \Omega_{\mathrm{D}, t}^{\mathrm{II}}\right]\right), \quad \lambda_{\mathrm{x}}>0,
\end{aligned}
$$

where $A_{t-1}$ denotes investors' aggregate holding of $\mathrm{FX}$ at the end of day $t-1$, and $\Omega_{\mathrm{D}, t}^{\mathrm{II}}$ denotes common information of dealers and the broker at the start of round II.

2. The trades initiated by dealer $d$ in round II are

$$
T_{d, t}^{\mathrm{II}}=\alpha_{\mathrm{Z}} Z_{d, t}^{\mathrm{I}}+\alpha_{\mathrm{A}} A_{t-1}
$$

and the customer orders received by dealer $d$ are in round I are

$$
Z_{d, t}^{\mathrm{I}}=(\beta / \mathrm{D}) Y_{t}+\varepsilon_{d, t}, \quad \beta<0
$$

where $\sum_{d=1}^{\mathrm{D}} \varepsilon_{d, t}=0$.

There are several noteworthy features of this equilibrium. First, consider its implications for the behavior of the spot exchange rate at the daily frequency:

$$
S_{t}^{\mathrm{III}}-S_{t-1}^{\mathrm{III}}=\frac{1}{r} V_{t}+\lambda_{\mathrm{X}}\left(X_{t}-\mathbb{E}\left[X_{t} \mid \Omega_{t}^{\mathrm{II}}\right]\right)
$$


Daily changes in the spot exchange rate are driven by shocks to dividends and unexpected interdealer order flow. The former reflect the effects of public news while the latter conveys information that was initially dispersed across investors and was then aggregated via trading in the FX market.

Dividend shocks play a familiar role in the determination of the spot rate. Realizations of $V_{t}$ are public information, and affect the forecasts of future dividends by all dealers and investors in exactly the same way. Consequently, it should come as no surprise that $V_{t}$ shocks are immediately reflected in the equilibrium spot rate. That said, it is important to remember that quotes are chosen optimally in this model, so $V_{t}$ shocks only affect the spot rate because dealers have an incentive to adjust their quotes once the value of $V_{t}$ is known (see equation 4a).

The role played by aggregate interdealer order flow in (7) is more complex. Notice that it is unexpected interdealer order flow that affects the exchange rate in (7). The reason is that dealers adjust their quotes between rounds II and III to account for the new information contained in aggregate interdealer order flow (see equation 4c). The customer orders received by each dealer reflect the difference between the desired and actual FX positions of individual investors. As such, they convey information to dealers about both the current income and the overnight positions of a subset of investors. This information is effectively shared between dealers via interdealer trading in round II. As a result, $X_{t}$ conveys information about aggregate income and the prior overnight FX position of all investors. Since the latter is already known to dealers, $X_{t}-\mathbb{E}\left[X_{t} \mid \Omega_{t}^{\mathrm{I}}\right]$ is proportional to the new information concerning income that is incorporated into the round III quote, $S_{t}^{\text {III }}$.

Of course, the mere fact that unexpected interdealer order flow conveys new information about aggregate income to dealers does not explain why $X_{t}-\mathbb{E}\left[X_{t} \mid \Omega_{t}^{\mathrm{I}}\right]$ appears in equation (7). For that we need to understand why dealers find it optimal to incorporate the new income information they learn into their common round III quote. In short, why is information on aggregate income, $Y_{t}$, relevant for the pricing of FX? The answer is quite simple. As in the actual market, dealers do not want to hold FX overnight - the risk of holding FX can be shared more efficiently by investors than by individual dealers. Consequently, each dealer's aim in round III is to quote a price that will induce investors to purchases all the FX currently held by dealers. In other words, the round III quote is chosen so that the excess overnight return expected by investors is such that they want to hold the entire existing stock of the FX. Obviously, this would not be possible unless all dealers can calculate what the existing stock of FX is. However, since investors' income is the only source of FX, the existing stock can be computed from the history of aggregate income. Thus, information on $Y_{t}$ is price-relevant because it reveals to dealers what aggregate overnight FX position investors must be induced to hold. In sum, interdealer order flow conveys information about the shift in the FX portfolios of investors needed to achieve efficient risk-sharing. This is the origin of the term, Portfolio Shifts.

Another important feature of the model concerns timing. As we noted above, public news concerning current and future dividends is immediately and fully incorporated into the spot exchange rate. By contrast, it takes time for the information concerning income to be reflected in the dealer quotes. The reason for the delay is important. Information about income is originally transmitted to dealers via the customer orders they receive in round I. Thus, each dealer has some information about $Y_{t}$ at the start of round II, but the information is imprecise. At this point, each dealer could choose to use their private information on $Y_{t}$ in setting their quote, but this is not optimal in the model's trading environment. Instead, their best strategy is to quote the same price as in round I (which is the same across all dealers and only a function of common round I information), because to do otherwise would expose the dealer to arbitrage trading losses. As a result, the equilibrium spot rate remains unchanged between rounds I and II even though dealers have 
information about aggregate income. The spot rate only incorporates this information when it becomes common knowledge among dealers. This process of information aggregation takes place via interdealer trading in round II. The best strategy of each dealer is to use their private information concerning income in determining the trade they wish to initiate with other dealers. It is for this reason that interdealer order flow provides information on aggregate income that becomes common knowledge to dealers by the start of round III.

\subsection{Empirical Evidence}

Empirical support for the PS model first came from regressions of the daily depreciation rate on interdealer order flow and the change in the nominal interest differential:.

$$
\Delta s_{t}=\beta_{1} X_{t}+\beta_{2} \Delta\left(r_{t}-\hat{r}_{t}\right)+\zeta_{t},
$$

where $\Delta s_{t} \equiv s_{t}-s_{t-1}$ is change in the log spot rate between the end of days $t-1$ and $t, r_{t}$ is the nominal dollar interest rate and $\hat{r}_{t}$ is the nominal non-dollar interest rate. These regressions are motivated by (7) with $\Delta s_{t}$ replacing $S_{t}^{\mathrm{III}}-S_{t-1}^{\mathrm{III}}$, and actual interdealer order flow, $X_{t}$, replacing $X_{t}-\mathbb{E}\left[X_{t} \mid \Omega_{t}^{\mathrm{II}}\right]$. The first of these substitutions makes the empirical specification comparable to standard macro models but has no significant effect on the estimation results; the second is motivated by the assumption that expected interdealer order flow is zero. The nominal interest differential is included to account for the arrival of public news.

Table 1 shows regression results for the DM/USD and JPY/USD spot rates between May 1 to August 31, 1996. The data on interdealer order flow comes from the Reuters Dealing 2000-1 system. This was the dominant platform for interdealer trading at the time, accounting for approximately 90 per cent of all "direct" dealer-to-dealer trades. The table reports the coefficient estimates and standard errors in parenthesis for five versions of equation (8).

Three features of the estimation results are particularly noteworthy:

1. The coefficient on order flow, $X_{t}$, is is correctly signed and significant, with $t$-statistics above 5 in versions I, II and IV of the equation for both the DM/USD and JPY/USD. The positive sign indicates that net dollar purchases - a positive $X_{t}$ - lead to a higher FX price for the Dollar. For perspective, the estimated value of 2.1 for the order flow coefficient in the DM/USD equation translates to $\$ 1$ billion of net purchases increasing the DM price of a dollar by 0.8 pfennigs.

2. Almost all the explanatory power in the regressions is due to order flow. In specifications III and $\mathrm{V}$ where order flow is omitted, the $R^{2}$ statistics are less than 1 percent in both the DM/USD and JPY/USD equations. Moreover, the explanatory power of order flow is extraordinarily high. The $R^{2}$ statistics of 64 percent and 45 percent for the DM and JPY equations that include order flow are an order of magnitude higher than those found in other exchange rate models.

3. The coefficient on the change in the interest differential is correctly signed, but is only significant in the JPY/USD equation. The positive sign arises in the sticky-price monetary model, for example, because an increase in the US interest rate requires an immediate dollar appreciation (i.e. an increase in JPY/USD) to make room for UIP-induced expected dollar depreciation. 


\begin{tabular}{|c|c|c|c|c|c|}
\hline & & & Regressors & & \\
\hline & & $X_{t}$ & $\Delta\left(r_{t}-\hat{r}_{t}\right)$ & $r_{t-1}-\hat{r}_{t-1}$ & $R^{2}$ \\
\hline \multirow[t]{5}{*}{$\overline{\mathrm{DM}} / \mathrm{USD}$} & $\mathrm{I}$ & $\begin{array}{l}2 \cdot 14^{* *} \\
(0 \cdot 29)\end{array}$ & $\begin{array}{c}0.51 \\
(0.26)\end{array}$ & & $0 \cdot 64$ \\
\hline & II & $\begin{array}{l}2 \cdot 15^{* *} \\
(0 \cdot 29)\end{array}$ & & & $0 \cdot 63$ \\
\hline & III & & $\begin{array}{c}0 \cdot 62 \\
(0 \cdot 77)\end{array}$ & & $0 \cdot 01$ \\
\hline & IV & $\begin{array}{l}2 \cdot 15^{* *} \\
(0 \cdot 29)\end{array}$ & & $\begin{array}{c}0.02^{*} \\
(0.01)\end{array}$ & $0 \cdot 64$ \\
\hline & V & & & $\begin{array}{c}0.02 \\
(0.02)\end{array}$ & $0 \cdot 00$ \\
\hline \multirow[t]{5}{*}{ JPY/USD } & I & $\begin{array}{c}2 \cdot 86^{* *} \\
(0 \cdot 36)\end{array}$ & $\begin{array}{l}2 \cdot 47^{* *} \\
(0.92)\end{array}$ & & $0 \cdot 46$ \\
\hline & II & $\begin{array}{l}2 \cdot 61^{* *} \\
(0 \cdot 36)\end{array}$ & & & $0 \cdot 40$ \\
\hline & III & & $\begin{array}{c}0 \cdot 57 \\
(1 \cdot 20)\end{array}$ & & $0 \cdot 00$ \\
\hline & IV & $\begin{array}{l}2 \cdot 78^{* *} \\
(0 \cdot 38)\end{array}$ & & $\begin{array}{c}0 \cdot 02^{*} \\
(0 \cdot 01)\end{array}$ & $0 \cdot 42$ \\
\hline & V & & & $\begin{array}{c}0.01 \\
(0.01)\end{array}$ & $0 \cdot 00$ \\
\hline
\end{tabular}

Notes: The table reports slope coefficients and standard errors. Statistical significance at the $5 \%$ and $1 \%$ level is denoted by $*$ and ${ }^{* *}$. Source: Evans and Lyons (2002)

Figure 2 provides further perspective on the results in Table 1. Here the solid plots shows the path of the spot exchange rate over the 4 month sample. The dashed plot shows cumulative order flow over the same period measured against the right hand axis. Cumulative order flow is simply the sum of daily order flow i.e. $X_{t}^{\infty}=\sum_{i=1}^{t} X_{i}$. If all variations in spot rates reflected quote revisions driven only by order flow, the daily change in the spot rate should be proportional to daily order flow; i.e., $S_{t}-S_{t-1}=\lambda_{\mathrm{X}} X_{t}$. Under these circumstances, $S_{t}=\lambda_{\mathrm{X}} X_{t}^{\infty}$ so the plots should coincide. Figure 2 shows that this in not quite the case. Nevertheless, it is hard not to be impressed by the close correspondence between exchange rates and order flow over this sample period.

Figure 2 highlights two further features of the relation between order flow and currency returns. First, although the equation in (8) is estimated at the daily frequency, the estimation results have implications for the behavior of spot rates over much longer periods. In particular, since there is almost no serial correlation in daily depreciation rates, the $k$-day change in the log spot rate is well-approximated by

$$
s_{t}-s_{t-k} \cong \beta_{1} X_{t}^{k}+\beta_{2} \Delta^{k}\left(r_{t}-\hat{r}_{t}\right)+\zeta_{t, k}
$$

where $X_{t}^{k}=\sum_{i=0}^{k-1} X_{t-i}$ denotes order flow during the past $k$ days, and $\beta_{2} \Delta^{k}\left(r_{t}-\hat{r}_{t}\right)+\zeta_{t, k}$ identifies the impact of public news arriving during the same period. Thus, the rate of depreciation over $k$ days will be well-approximated by the cumulative effects of order flow, $X_{t, k}$, and the arrival of public news. Figure 2 


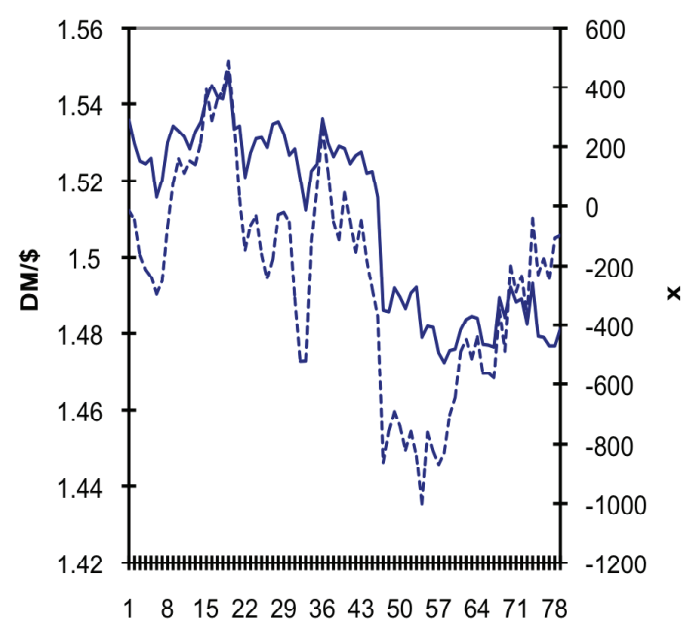

$\mathrm{DM} / \mathrm{USD}$

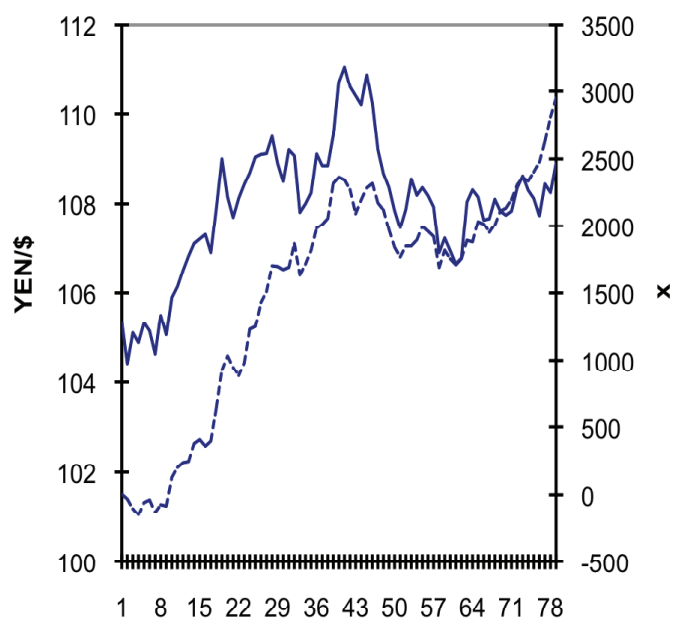

JPY/USD

Figure 2: Exchange Rates (solid) and Cumulative Order Flow (dashed). Source: Evans and Lyons (2002)

shows that there are many instances where $s_{t}-s_{t-k} \cong \beta_{1} X_{t}^{k}$ for significant horizons $k$.

The second feature concerns the dynamics of order flow. The plots of cumulative order flow in Figure 2 display no significant mean reversion because there is no detectable serial correlation in daily order flows. This feature of the data is important because the empirical specification in (8) assumes that realizations of daily order flow represent news to dealers. Thus daily order flow must be serially uncorrelated if the results in Table 1 are to be consistent with the predictions of the PS model.

The estimates in Table 1 are representative of those obtained in a large number of studies. The strong empirical link between interdealer order flows and daily changes in the spot exchange rate extends across different currencies, different time periods and over horizons ranging from a few minutes to several weeks. The results also extend to customer order flow. Recall that in the PS model interdealer order flow conveys price-relevant information to dealers which initially entered the market in dispersed form via the customer orders received by individual dealers. Consequently, we should expect to find that customer order flows also have explanatory power in accounting for exchange rate changes. This is indeed the case. Customer flows disaggregated by customer type have more explanatorily power for exchange rate changes than the aggregate flows received by individual banks. At the daily horizon, disaggregated flows account for less of the variation in exchange rate changes than aggregate interdealer order flows, but the explanatory power of customer and dealers flows are comparable over horizons of one to several weeks.

\section{From Micro to Macro}

Microstructure exchange-rate models, such as the PS model, are silent on the potential links between the macro economy and order flows because they focus on the details of currency trading between different market participants rather than the macroeconomic factors that ultimately drive the individual trades. To 
address this deficiency, recent research focuses on the role order flow plays in conveying macro information to the foreign exchange market. This research builds on two central ideas: First, only some of the macro information relevant for the current spot rate is publicly known at any point in time. Other information is present in the economy, but it exists in a dispersed microeconomic form. The second idea relates to determination of the spot rate. Since it literally is the price of foreign currency quoted by foreign exchange dealers, the spot rate can only embed information that is known to dealers. Consequently, the spot rate will only reflect dispersed information once it has been assimilated by dealers - a process that takes place via trading. Below I provide an overview of a micro-based model that incorporates these ideas and discuss its empirical implications.

\subsection{A Micro-Based Model}

The economy comprises two countries populated by a continuum of risk-averse agents, indexed by $n \in[0,1]$; and D risk-averse dealers who act as marketmakers in the spot FX market. I will refer to the home and foreign countries as the US and Europe, so the log spot exchange rate, $s_{t}$, denotes the dollar price of euros. The only other actors in the model are the central banks (i.e., The Federal Reserve (FED) and the European Central Bank (ECB)), who conduct monetary policy by setting short-term nominal interest rates, $r_{t}$ and $\hat{r}_{t}$.

\subsubsection{Overview}

The model focuses on the currency trades between agents and dealers at a weekly frequency. At the start of week $t$, all dealers and agents observe a data release that provides information on the state of the economy several weeks earlier. Let $z_{t}$ denote a vector of variables that completely describe the state of the macroeconomy in week $t$. This vector contains variables that are directly observable, like short-term interest rates, and others that only become publicly known with a lag. Each agent $n$ also receives private information concerning his or her current microeconomic environment: $z_{t}^{n}=z_{t}+v_{t}^{n}$, where $v_{t}^{n}=\left[v_{i, t}^{n}\right]$ is a vector of agent-specific shocks with the property that $\int_{0}^{1} v_{i, t}^{n} d n=0$ for all $i$. Notice that the $z_{t}^{n}$ vector represents dispersed private information about macro conditions.

Next, all dealers quote a log USD/EUR spot price equal to

$$
s_{t}=\mathbb{E}_{t}^{\mathrm{D}} s_{t+1}+\hat{r}_{t}-r_{t}-\delta_{t},
$$

where $\mathbb{E}_{t}^{\mathrm{D}}$ denotes expectations conditioned on the common information available to all dealers at the start of week $t$. This information set includes the histories of interest rates and data releases, and the order flows from earlier currency trading. The foreign exchange risk premium, $\delta_{t}$, is chosen by dealers to share risk efficiently across the FX market.

Macroeconomic conditions affect dealers quotes in (9) via their affects on current monetary policy (i.e., $\left.\hat{r}_{t}-r_{t}\right)$, and via $\mathbb{E}_{t}^{\mathrm{D}} s_{t+1}$ insofar as these expectations are affected by dealers' forecasts for the future interest differential. These forecasts are assumed to incorporate a view on how central banks react to changes in the macroeconomy. In particular,

$$
\mathbb{E}_{t}^{\mathrm{D}}\left(\hat{r}_{t+i}-r_{t+i}\right)=\left(1+\gamma_{\pi}\right) \mathbb{E}_{t}^{\mathrm{D}}\left(\Delta \hat{p}_{t+1+i}-\Delta p_{t+1+i}\right)+\gamma_{y} \mathbb{E}_{t}^{\mathrm{D}}\left(\hat{y}_{t+i}-y_{t+i}\right)-\gamma_{\varepsilon} \mathbb{E}_{t}^{\mathrm{D}} \varepsilon_{t+i},
$$

for $i>0$, where $\gamma_{\pi}, \gamma_{y}$ and $\gamma_{\varepsilon}$ are positive coefficients. Equation (10) implies that dealers expect the future differential between euro and dollar rates to be higher when: (i) the future difference between EU and US 
inflation, $\Delta \hat{p}_{t+1}-\Delta p_{t+1}$, is higher; (ii) the difference between the EU and US output gaps, $\hat{y}_{t}-y_{t}$, widens; or (iii) when the real exchange rate, $\varepsilon_{t} \equiv s_{t}+\hat{p}_{t}-p_{t}$, depreciates. These expectations are consistent with widely-accepted views about how central banks react to changing macroeconomic conditions.

Dealers stand ready to fill the foreign currency orders of agents at their quoted price of $s_{t}$. Once agent $n \in[0,1]$ observes $s_{t}$, they place their currency order with a dealer to fill their desired demand for euros. The order flow for euros from agent $n$ is therefore $\alpha_{t}^{n}-\alpha_{t-1}^{n}$ where $\alpha_{t}^{n}$ denotes the week- $t$ demand by agent $n$. This demand is given by

$$
\alpha_{t}^{n}=\alpha_{s}\left(\mathbb{E}_{t}^{n} \Delta s_{t+1}+\hat{r}_{t}-r_{t}\right)+h_{t}^{n},
$$

where $\alpha_{s}>0$ and $\mathbb{E}_{t}^{n}$ denotes expectations conditioned on the information available to agent $n$ after the spot rate is quoted at the start of week $t$. The demand for euros depends on the log excess return expected by the agent and a hedging term, $h_{t}^{n}$, that represents the influence of all other factors. Without loss of generality, we may assume that $h_{t}^{n}=\alpha_{z} z_{t}^{n}$, for some vector $\alpha_{z}$, where the vector $z_{t}^{n}$ describes the microeconomic environment of agent $n$. The aggregate demand for euros by agents is thus given by

$$
\alpha_{t}=\int_{0}^{1} \alpha_{t}^{n} d n=\alpha_{s}\left(\overline{\mathbb{E}}_{t}^{n} s_{t+1}-s_{t}+\hat{r}_{t}-r_{t}\right)+h_{t},
$$

where $h_{t}=\int_{0}^{1} h_{t}^{n} d n$ is the aggregate hedging demand and $\overline{\mathbb{E}}_{t}^{n}$ denotes the average of agents' expectations: $\overline{\mathbb{E}}_{t}^{n} s_{t+1}=\int_{0}^{1} \mathbb{E}_{t}^{n} s_{t+1} d n$. Notice that $h_{t}=\alpha_{z} \int_{0}^{1} z_{t}^{n} d n=\alpha_{z} z_{t}$, so the aggregate hedging demand depends on the state of the macroeconomy, $z_{t}$.

For the remainder of the week, dealers trade among themselves. The model abstracts from the details of interdealer trade. Instead, consistent with the PS model, it assumes that all dealers learn the aggregate order flow that resulted from the earlier week $-t$ trades between agents and dealers. This order flow is determined by

$$
X_{t+1}=\alpha_{t}-\alpha_{t-1}
$$

Notice that order flow is drriven by changes in the average of agents' expectations concerning future excess returns and the change in the aggregate hedging demand. (The $t+1$ subscript makes clear that order flow is only observed by dealers at the end of week- $t$ trading.)

\subsubsection{Equilibrium}

An equilibrium in this model comprises a sequence of spot rates and market-clearing order flows that support and are supported by the endogenous evolution of dealers' common information and agents' private information. Recall that dealers and agents observe current interest rates and the data releases containing information on past macroeconomic conditions. Dealers also obtain information from their observations on order flow that is endogenously determined by the trading decisions of agents. Similarly, each agent observes the endogenously determined spot rate together with their microeconomic environment.

Solving for equilibrium spot rates and order flows is complex. But, so long as public information about the current state of macroeconomy is incomplete, equilibrium order flows provide dealers with information about the macroeconomy that they will embed in the spot rate quotes; information that was initially dispersed across agents as their observations on $z_{t}^{n}$.

In equilibrium, the spot rate quoted by dealers satisfies equation (9) subject to the risk-sharing restriction 
that identifies the risk premium, and dealers' expectations concerning future interest rates in (10):

$$
s_{t}=\left(\hat{r}_{t}-r_{t}\right)+\mathbb{E}_{t}^{\mathrm{D}} \sum_{i=1}^{\infty} \rho^{i} f_{t+i}-\mathbb{E}_{t}^{\mathrm{D}} \sum_{i=0}^{\infty} \rho^{i} \delta_{t+i}
$$

with $\rho \equiv 1 /\left(1+\gamma_{\varepsilon}\right)<1$, where

$$
f_{t}=\left(1+\gamma_{\pi}\right)\left(\Delta \hat{p}_{t+1}-\Delta p_{t+1}\right)+\gamma_{y}\left(\hat{y}_{t}-y_{t}\right)+\left(\frac{1-\rho}{\rho}\right)\left(p_{t}-\hat{p}_{t}\right) .
$$

The three terms on the right of equation (14) identify different factors affecting the log spot rate dealers quote at the start of week $t$. First, the current stance of monetary policy affects dealers' quotes via the interest differential, $\hat{r}_{t}-r_{t}$, because it directly contributes to the payoff from holding euros until week $t+1$. Second, dealers are concerned with the future course of macro fundamentals, $f_{t}$. This term embodies dealers' expectations of how central banks will react to macroeconomic conditions when setting future interest rates. The third factor arises from risk-sharing between dealers and agents as represented by the present and expected future values of the risk premium, $\delta_{t}$. These values are determined by

$$
\delta_{t}=\mathbb{E}_{t}^{\mathrm{D}}\left[s_{t+1}^{e}-\frac{1}{\alpha_{s}} h_{t}\right]
$$

where $s_{t+1}^{e}=s_{t+1}-\overline{\mathbb{E}}_{t}^{n} s_{t+1}$. Intuitively, dealers stabilize their euro holdings by lowering the risk premium when they anticipate a rise in the aggregate hedging demand for euros because the implied fall in the excess return agents' expect will offset their desire to accumulate larger euro holdings. Dealers also reduce the risk premium to offset agents' desire to accumulate larger euro holdings when they are viewed as being too optimistic (on average) about the future spot rate; i.e., when $\mathbb{E}_{t}^{\mathrm{D}} s_{t+1}<\mathbb{E}_{t}^{\mathrm{D}} \overline{\mathbb{E}}_{t}^{n} s_{t+1}$.

In equilibrium, the aggregate order flow dealers observe at the end of week- $t$ trading reflects the change in aggregate demand for foreign currency by agents between weeks $t-1$ and $t: X_{t+1}=\alpha_{t}-\alpha_{t-1}$. Since dealers know the history of order flow and $\alpha_{t-1}=\sum_{i=0}^{\infty} X_{t-i}$ by market clearing, $\alpha_{t-1} \in \Omega_{t}^{\mathrm{D}}$. Consequently, unexpected order flow from week- $t$ trading can be written as

$$
X_{t+1}-\mathbb{E}_{t}^{\mathrm{D}} X_{t+1}=\alpha_{z}\left(z_{t}-\mathbb{E}_{t}^{\mathrm{D}} z_{t}\right)+\alpha_{s}\left(\overline{\mathbb{E}}_{t}^{n} s_{t+1}-\mathbb{E}_{t}^{\mathrm{D}}\left[\overline{\mathbb{E}}_{t}^{n} s_{t+1}\right]\right) .
$$

Thus order flow contributes new information about the current macroeconomic conditions, $z_{t}$, via the aggregate hedging demand, $h_{t}-\mathbb{E}_{t}^{\mathrm{D}} h_{t}=\alpha_{z}\left(z_{t}-\mathbb{E}_{t}^{\mathrm{D}} z_{t}\right)$, and about the average of agents' spot rate forecasts, $\overline{\mathbb{E}}_{t}^{n} s_{t+1}$. These forecasts will embed agents private expectations concerning future interest rates, and the macroeconomic conditions that affect monetary policy.

\subsection{Empirical Evidence}

We can use equations (14) - (16) to examine the models' empirical implications. Consider, first, the behavior or excess returns, $e r_{t+1}=\Delta s_{t+1}+\hat{r}_{t}-r_{t}$. Equation (9) implies that, $e r_{t+1}=\delta_{t}+s_{t+1}-\mathbb{E}_{t}^{\mathrm{D}} s_{t+1}$, so substituting for the dealers' forecast errors gives

$$
e r_{t+1}=\delta_{t}+\left(1-\mathbb{E}_{t}^{\mathrm{D}}\right)\left(\hat{r}_{t+1}-r_{t+1}\right)+\left(\mathbb{E}_{t+1}^{\mathrm{D}}-\mathbb{E}_{t}^{\mathrm{D}}\right) \sum_{i=2}^{\infty} \rho^{i-1}\left(f_{t+i}-\frac{1}{\rho} s_{t+i}^{e}-\frac{1}{\rho \alpha_{s}} h_{t+i-1}\right) .
$$

Notice that the second and third terms depend on the flow of information reaching all dealers between the start of weeks $t$ and $t+1$. This information flow comprises observations on current interest rates, data 
releases on past macro variables, and order flow from week $-t$ trading, $X_{t+1}$. Equation (17) implies that any of these information sources will affect the excess returns insofar as they convey new information on current monetary policy, future fundamentals and the risk premia. Thus the model attributes the strong correlation between foreign currency returns and order flows observed in the data to the important role order flow plays in this information transmission process.

We can examine this implication of the model with a two step regression procedure. In the first step we regress a macro variable $\varkappa_{t}$ on the cumulation of current and past order flows, $X_{t+1}^{\infty}=\sum_{i=0}^{\infty} X_{t+1-i}$. Under efficient risk-sharing $X_{t+1}^{\infty}=X_{t+1}-\mathbb{E}_{t}^{\mathrm{D}} X_{t+1}$, so the regression coefficient on $X_{t+1}^{\infty}$ should be statistically significant if unexpected order flow from week $-t$ trading contains information about macro variable $\varkappa_{t}$. In the second step we regress excess returns on the predicted values from the first stage. The estimated slope coefficient in this regression should be statistically significant when the information concerning $\varkappa_{t}$ conveyed by order flow is price-relevant.

Table 2 reproduces the results from estimating the second stage regression as reported in Evans (2010). The dependent variable is the excess return on the USD/EUR spot over a 4 week horizon. The dependent variables in Panel A are the predicted values from regressions of GDP growth, CPI inflation and M1 growth in the US and Germany on cumulated customer order flows for euros received by Citibank. In panel B the dependent variables are the differences between the predicted values for the German and US macro variables. The regressions are estimated at the weekly frequency in data from January 1993 to June 1999.

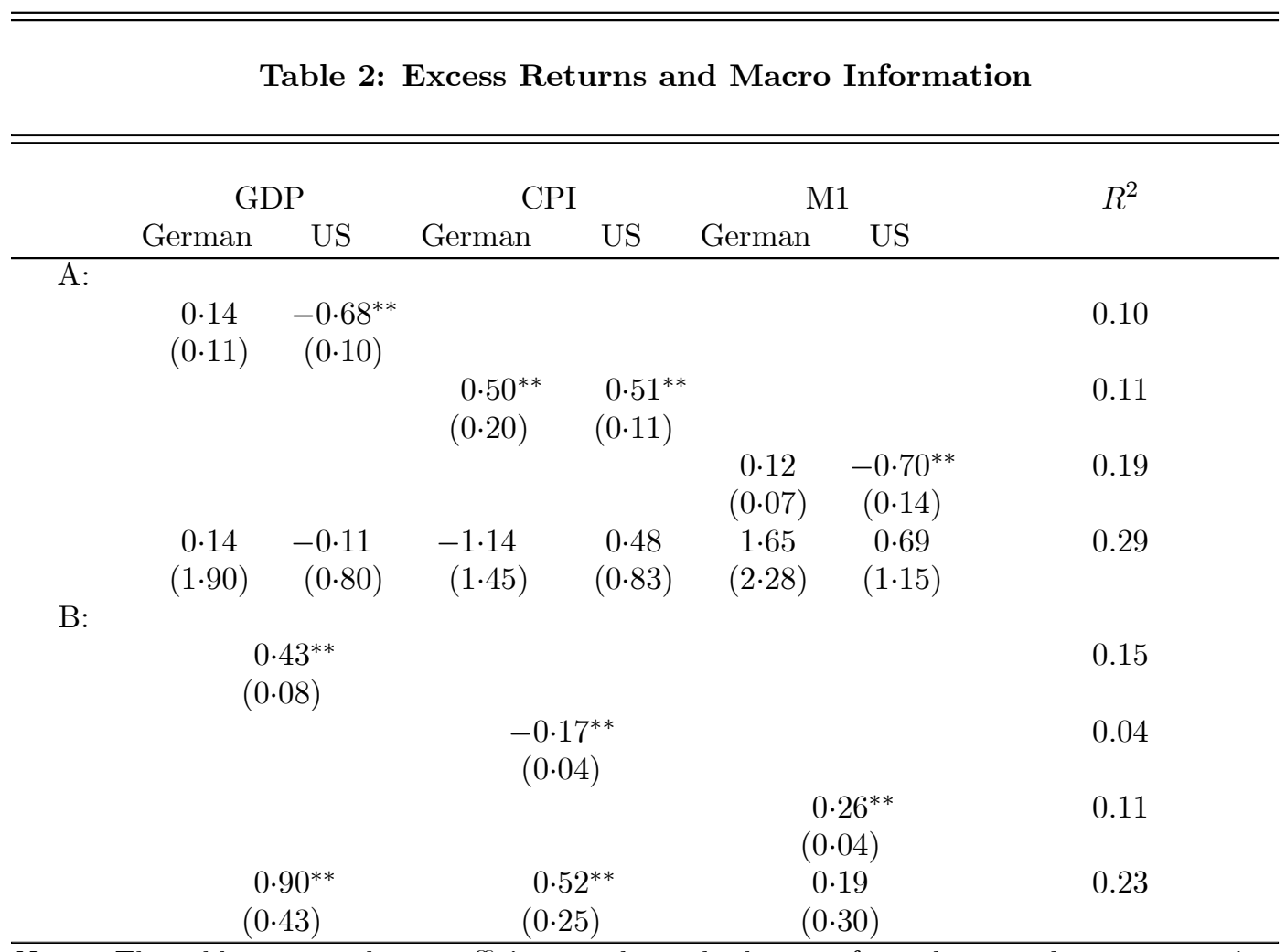

Notes: The table reports slope coefficients and standard errors from the second stage regression using the predicted values for the variables listed at the head of each column. Estimates are calculated at the weekly frequency. The standard errors correct for heteroskedasticity. and an MA(3) error process. Statistical significance at the $5 \%$ and $1 \%$ level is denoted by $*$ and **. Source: Evans (2010) 
The results in Table 2 provide strong support for the idea that order flows convey price-relevant information about GDP, prices and the money stock. The slope coefficients for German inflation, US GDP growth, inflation and monetary growth are all significant at the one percent level. Insofar as spot rates reflect the difference between US and EU monetary policy, order flows should carry more price-relevant information about the difference in macroeconomic conditions between countries. This seems to be the case. As Panel B shows, the slope coefficients on the predicted values for GDP growth and inflation are highly significant.

The model also holds implications for the links between the dynamics of spot rates, order flows and the future evolution of the macro economy. In particular, (14) implies that

where

$$
\begin{gathered}
s_{t}=s_{t}^{*}+\frac{1}{1-\rho} \mathbb{E}_{t}^{\mathrm{D}} \sum_{i=1}^{\infty} \rho_{t+i}^{i}\left(\Delta f_{t+i}-\Delta \delta_{t+i}\right) \\
s_{t}^{*}=\left(\hat{r}_{t}-r_{t}\right)+\frac{\rho}{1-\rho} \mathbb{E}_{t}^{\mathrm{D}} f_{t}-\frac{1}{1-\rho} \delta_{t} .
\end{gathered}
$$

This equation splits the factors affecting dealers' spot rate quotes into two terms. Current conditions, such as interest rats and dealer's estimates of week $-t$ fundamentals affect dealers' quotes via $s_{t}^{*}$. Expected changes in future conditions (i.e., inflation, the output gaps and the risk premium), affect the spot rate via the second term. It is this term that provides the link between spot rates, order flows and the future path of macro variables.

Equation (18) implies that $s_{t}-s_{t}^{*}$ will have forecasting power for any future macro variable, $\varkappa_{t+\tau}$, if dealers expectations, $\mathbb{E}_{t}^{\mathrm{D}} \varkappa_{t+\tau}$, are correlated with $\mathbb{E}_{t}^{\mathrm{D}}\left(\Delta f_{t+i}-\Delta \delta_{t+i}\right)$. Intuitively, dealers will raise their spot rate quotes relative to $s_{t}^{*}$ when their forecasts of future changes in fundamentals increase, so if these forecast are correlated with $\mathbb{E}_{t}^{\mathrm{D}} \varkappa_{t+\tau}$, variations in $s_{t}-s_{t}^{*}$ will have forecast power for $\varkappa_{t+\tau}$. Equation (18) also implies that order flow should generally have incremental forecasting power for future macro variables beyond that contained in $s_{t}-s_{t}^{*}$. To understand why, consider the following identity:

$$
\varkappa_{t+\tau}=\mathbb{E}_{t}^{\mathrm{D}} \varkappa_{t+\tau}+\left(\mathbb{E}_{t+1}^{\mathrm{D}}-\mathbb{E}_{t}^{\mathrm{D}}\right) \varkappa_{t+\tau}+\left(1-\mathbb{E}_{t+1}^{\mathrm{D}}\right) \varkappa_{t+\tau}
$$

The first term on the right identifies dealers' expectations concerning $\varkappa_{t+\tau}$ based on the information they use to quote spot rates at the start of week $t$. The second term identifies the revision in dealers' forecasts between the start of weeks $t$ and $t+1$. The incremental forecasting power of order flow comes from this term, which, by construction, is uncorrelated with $\mathbb{E}_{t}^{\mathrm{D}} \varkappa_{t+\tau}$. In particular, any information conveyed by $X_{t+1}-\mathbb{E}_{t}^{\mathrm{D}} X_{t+1}$ concerning $\varkappa_{t+\tau}$ will lead dealers to revise their forecasts of $\varkappa_{t+\tau}$.

This implication of the model can be examined with a regression of $\varkappa_{t+\tau}$ on $s_{t}$ and other variables known to dealers at the start of week $t$, and unexpected order flow from trades between dealers and agents during week $t$. The coefficients on the order flows should be statistically significant if they have incremental forecasting power for the macro variable in question. Furthermore, the portion of the predicted value for $\varkappa_{t+\tau}$ attributable to the order flows should make a significant contribution to the variance of $\varkappa_{t+\tau}$ if the information conveyed by the order flows is economically significant.

Figure 3 reproduces the plots from Evans and Lyons (2009) that show the variance contributions of the order flows together with 95 percent confidence bands for the six macro variables considered in Table 2 for 


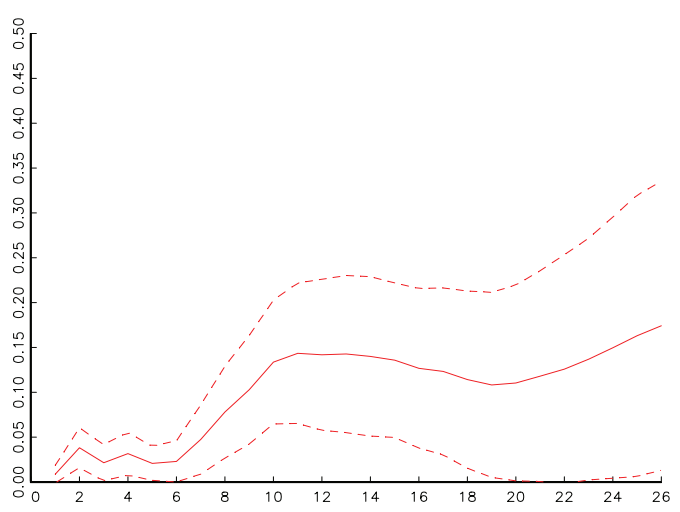

A: US GDP Growth

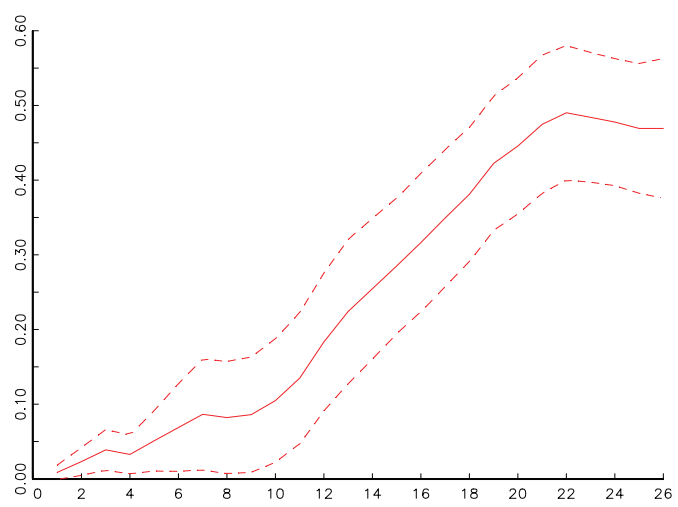

C: US Inflation

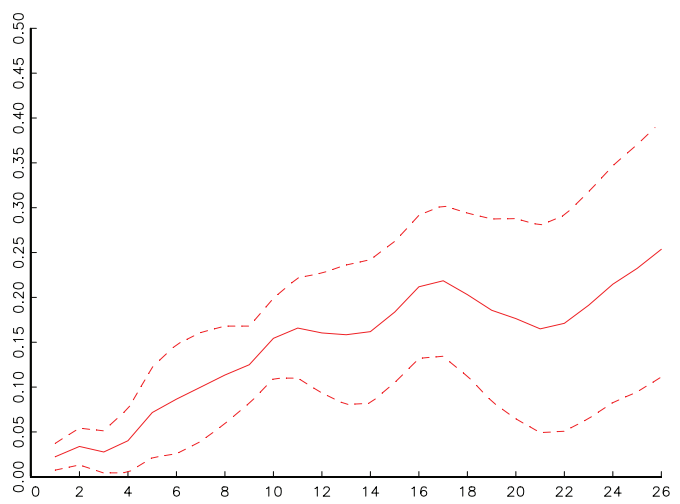

E: US M1 Growth

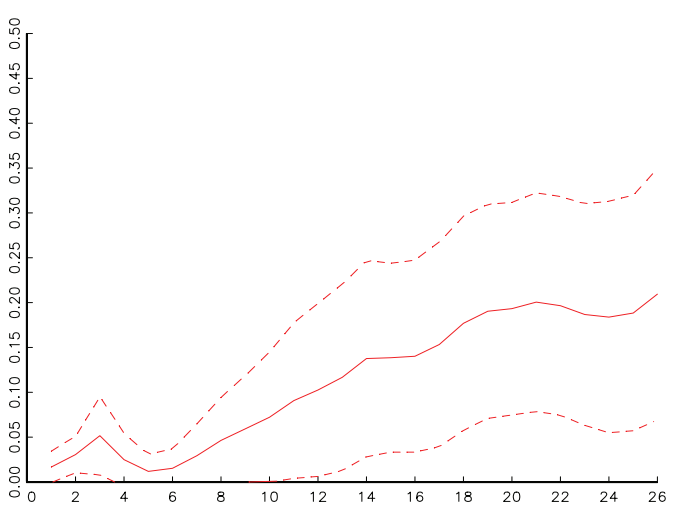

B: German GDP Growth

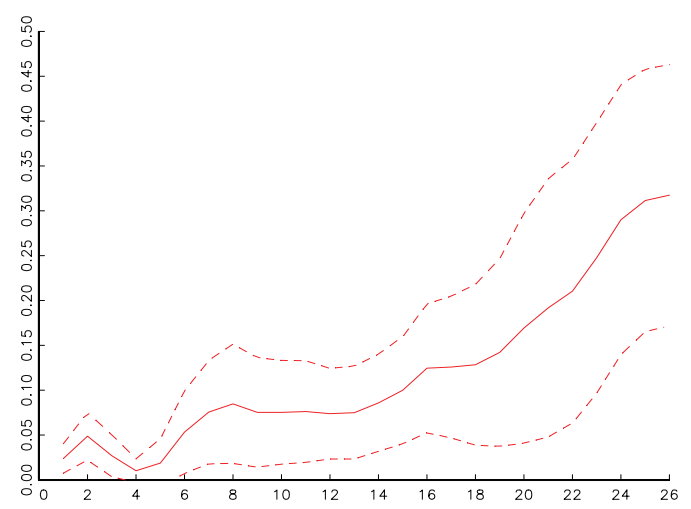

D: German Inflation

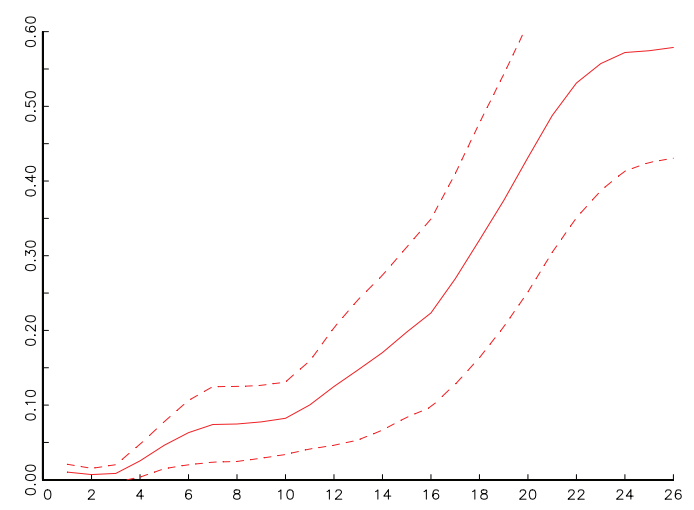

F: German M1 Growth

Figure 3: Estimated Contribution of Order Flows to the Variance of Future GDP growth, Inflation and M1 growth by forecasting horizon, $\tau$, measured in weeks. Dashed lines denote $95 \%$ percent confidence bands computed as $\pm 1.96 \hat{\sigma}$, where $\hat{\sigma}$ is the standard error of the estimated contribution. Source: Evans and Lyons (2009)

horizons $\tau=1, . ., 26$ weeks. In five of the six cases, the contributions rise steadily with the horizon and are quite sizable beyond one quarter. The exception is US GDP growth, where the contribution remains around 15 percent from the quarterly horizon onward. These plots clearly show that order flows have considerable forecasting power for the future flows of information concerning GDP growth, inflation and M1 growth at all but the shortest horizons. Clearly, then, these order flows are carrying significant information on future macroeconomic conditions. 


\section{Micro Perspectives on Exchange-Rate Puzzles}

Micro-based models provide a new perspective on some long-standing puzzles concerning the behavior of exchange rates over horizons ranging from a few minutes to several years. Below I discuss the micro-based perspective on the Disconnect and News puzzles.

\subsection{The Disconnect Puzzle}

The exchange rate Disconnect Puzzle refers to the observation that most short- and medium-term variations in spot exchange rates appear to be unrelated to changes in current or expected future macro variables that should identify fundamentals. In other words, exchange-rate variations appear essentially disconnected from changing macroeconomic conditions over horizons up to several years.

To gain a micro-based perspective on this puzzle, consider the week-by-week variations in the log spot rate implied by the micro-based the model:

$$
\begin{aligned}
\Delta s_{t+1} & =r_{t}-\hat{r}_{t}+\delta_{t} \\
& +\left(1-\mathbb{E}_{t}^{\mathrm{D}}\right)\left(\hat{r}_{t+1}-r_{t+1}\right)+\frac{1}{1-\rho}\left(\mathbb{E}_{t+1}^{\mathrm{D}}-\mathbb{E}_{t}^{\mathrm{D}}\right)\left(\rho f_{t+1}-\delta_{t+1}\right) \\
& +\frac{1}{1-\rho}\left(\mathbb{E}_{t+1}^{\mathrm{D}}-\mathbb{E}_{t}^{\mathrm{D}}\right) \sum_{i=2}^{\infty} \rho^{i-1}\left(\Delta f_{t+i}-\Delta \delta_{t+i}\right) .
\end{aligned}
$$

It is well-established that interest differentials and other macro variables have very little forecasting power for short- and medium-term depreciation rates (see, e.g., Table 1). Thus, as a purely empirical matter, there appears to be little prospect to establishing the link between short-term variations in spot rates and macro variables known at the start of week $t$ via interest rates and the risk premium terms; $r_{t}, \hat{r}_{t}$ and $\delta_{t}$. Instead, we must focus our attention on the terms in the second and third lines of equation (20). The two terms in the second line identify the effects of new information concerning current macroeconomic conditions, i.e., conditions at the start of week $t+1$. The first identifies the effect of unanticipated changes in short-term interest rates. In practice, central banks change interest rates relatively rarely; and when they do, they often communicate their intentions beforehand so as not to put undue stress on the financial system. As a result, unanticipated changes in short-term interest are not an important source of high-frequency spot rate dynamics over long time spans. This leaves the terms involving macro fundamentals, $f_{t}$, and the risk premium, $\delta_{t}$. It is in the identification of these terms that the macro and micro-based perspectives differ.

Macro models typically assume that uncovered interest parity holds (or the risk premium is constant) so their focus is on the terms involving fundamentals. If the current state of the macroeconomy is known to all market participants (including dealers) as macro models assume, these terms become

$$
\frac{\rho}{1-\rho}\left(1-\mathbb{E}_{t}^{\mathrm{D}}\right) \Delta f_{t+1}+\frac{1}{1-\rho}\left(\mathbb{E}_{t+1}^{\mathrm{D}}-\mathbb{E}_{t}^{\mathrm{D}}\right) \sum_{i=2}^{\infty} \rho^{i-1} \Delta f_{t+i} .
$$

Under normal circumstances, the first term in this expression should be close to zero because macro fundamentals are unlikely to change significantly during a week. Thus, from a macro perspective, the lion's share of the link between macro fundamentals and high-frequency spot rate dynamics must be attributable to the second term (i.e., the revisions in forecasts about future changes in fundamentals, $\Delta f_{t+i}$ for $i>1$ ). However, the time series properties of the macro variables used to construct fundamentals, $\hat{f}_{t}$, imply very little variation in the estimates of the present value of future changes in $\hat{f}_{t}$. As a result, estimates of the 
second term in (21) using $\hat{f}_{t}$ account for roughly five percent of the variance of the depreciation rates at the monthly and quarterly horizons. By this metric, the high frequency movements in exchange rates appear largely disconnected from measured macro fundamentals.

The micro-based perspective on the disconnect puzzle is rather different because all the terms on the right of equation (20) involving fundamentals and the risk premium could link short-term variations in spot rates to the macroeconomy.

Consider the terms involving fundamentals. When the weekly changes in fundamentals are negligible (i.e., $\Delta f_{t+1} \cong 0$ ), the terms involving fundamentals are well-approximated by

$$
\frac{\rho}{1-\rho}\left(\mathbb{E}_{t+1}^{\mathrm{D}}-\mathbb{E}_{t}^{\mathrm{D}}\right) f_{t}+\frac{1}{1-\rho}\left(\mathbb{E}_{t+1}^{\mathrm{D}}-\mathbb{E}_{t}^{\mathrm{D}}\right) \sum_{i=2}^{\infty} \rho^{i-1} \Delta f_{t+i} .
$$

From a micro-based perspective, dealers are not assumed to have contemporaneous information on the macro variables that comprise fundamentals, so the first term in (22) can differ from zero (because dealers are learning about past macroeconomic conditions that drive $f_{t}$ ). Moreover, this term appears to be significant empirically. As Table 2 showed, order flow from week- $t$ trading convey significant new information concerning current GDP, prices and money stocks, that is incorporated into spot rates. Thus, some of the high frequency behavior of exchange rates reflects the flow of new information reaching dealers concerning the slowly evolving state of the macroeconomy.

The micro-based model also provides a new perspective on the second term in (22). Figure 2 shows that order flows have significant incremental forecasting power for future macro variables beyond that contained in current spot rates and other variables. Insofar as dealers use the information in these flows to revise their expectations, estimates of the second term in (22) based on macro data will understate the degree to which revisions in dealers expectations concerning future changes in fundamentals contribute to spot rate dynamics.

In sum the micro-based evidence compliments rather than contradicts the empirical evidence derived from macro models on the link between spot rates and the macroeconomy. By concentrating on the flows of information available to dealers, micro-based models point to the role of order flow as a carrier of macroeconomic information. This information appears useful in revising forecasts of future changes in macro fundamentals, the channel emphasized by macro models. It also appears useful in revising dealer's estimates of current macroeconomic conditions.

\subsection{The News Puzzle}

One striking symptom of the Disconnect Puzzle shows up in research on the effects of macro data releases. This research shows that spot rates react to data releases in a manner consistent with the predictions of standard macro models, but the releases account for less than one percent of the total variance in spot rates. In other words, most of the volatility in spot rates takes place during periods when there are no data releases.

This represents a puzzle from the macro perspective. Macro exchange-rate models predict that spot rates will respond immediately to the public release of macro data if it induces: (i) a change in current interest rates and/or the risk premium, and/or (ii) a revision in expectations concerning future fundamentals and/or the risk premia. The models do not predict that spot rates will respond to all data releases, or even to previously unexpected releases. Nor do they predict the size or direction of the spot rate's response to particular releases. They do, however, rule out the possibility that the spot rate's response is delayed. 
Consequently, only the variations in spot rates immediately following a data release are attributable to the effects of macro news.

Micro-based models provide several new perspectives on how the release of macro data affects the behavior of spot rates. First, data releases will only affect spot rates insofar as they induce dealers to revise their spot rate quotes. Since data releases relate to past macroeconomic conditions, it is possible that most of the information they contain was already known to dealers from their past observations on order flows. A data release may contain new information about the macroeconomy relative to prior public information, but it may contain little incremental information to dealers. Consequently, the initial reaction of the spot rate to a data release will be smaller than if order flow were uninformative about current macroeconomic conditions.

The immediate reaction of the spot rate to a data release may also be muted for other reasons. Although data releases are simultaneously observed by dealers and other agents, there may not be unanimous agreement on their implications for the value of foreign currency. For example, two firms may interpret the same announcement on last quarter's GDP as having different implications for future interest rates. When a data release contains imprecise but price-relevant information, dealers will immediately adjust their spot rate quotes to accommodate the new information on fundamentals and the risk of providing liquidity to the market. These effects can offset one-another, so that immediate impact of the data release on the spot rate is muted.

Differing interpretations about the implications of a data release can also lead to changes in dealers spot rate quotes for some time after the release takes place. Some of these changes reflect the risk premia that was embedded in dealers original quotes. Others reflect the effects of order flow on dealers' quotes as market participants reach a consensus about the price-implications of the release. For example, suppose the data release represents good news for the dollar, but there is diverse opinion on how far the dollar price of euros should fall. Under these circumstances, the initial fall in the USD/EUR spot rate may be viewed as too large by some market participants and too small by others. Those who view the fall as too small will place orders to sell the euro, while those who view the fall as too large will place orders to buy. In aggregate, the balance of these trades represents the order flow that dealers use to further revise their spot rate quotes. In particular, positive (negative) order flow for the euro signals that the initial USD/EUR spot rate was below (above) the balance of opinion among market participants concerning the implications of the data release.

Empirical research supports this micro-based perspective on the effects of data releases. Studies of intraday data indicate that data releases affect spot rates directly and indirectly via induced order flows. Furthermore, order flows appear to carry more price-relevant information that is incorporated into spot rates following macro data releases than at other times. At the daily frequency, more than one third of the total variance in spot rate changes can be related to the direct and indirect effects of macro data releases and other news sources. The indirect effects of news working via order flow contribute approximately 60 percent more to the variance of spot rate changes than do the direct effects. In short, micro-based research shows that data releases play a more important role in the determination of spot rates than was indicated by earlier research based on macro models.

\section{Conclusion}

Although micro-based research over the past has made some significant progress towards providing exchangerate models with empirically relevant microfoundations, much remains to be done. The models developed to date are designed to study the behavior major currency markets with lots of trading activity, such as the 
USD/EUR and USD/JPY markets. They are less well-suited for studying the behavior of exchange rates between other currencies that trade with much less liquidity. Since the currencies of most counties fall into this category, adapting micro-based models to these markets is an important priority for future research. The models also need to expand their focus beyond the spot markets. Trade in forward contracts, swaps and other exchange-rate derivatives account for a large share of FX trading activity, but this portion of the FX market has yet to be studied from a micro-based perspective. This will surely change as data from electronic trading systems becomes more accessible to researchers.

\section{Further Reading}

Evans, M. D. D. (2010), Exchange Rate Dynamics, Princeton University Press, forthcoming.

Evans, M. D. D. (2010). Order flows and the exchange rate disconnect puzzle. Journal of International Economics 80(1), $58-71$.

Evans, M. D. D. and R. K. Lyons (2002). Order flow and exchange rate dynamics. Journal of Political Economy 110(1), $170-180$.

Evans, M. D. D. and R. K. Lyons (2009). Forecasting exchange rate fundamentals with order flow. Working paper, Georgetown University.

Lyons, R. K. (2001). The Microstructure Approach to Exchange Rates. Cambridge, MA: MIT Press.

Osler, C. L. (2008). Springer Encyclopedia of Complexity and System Science., Chapter Foreign Exchange Microstructure: A Survey.

Sarno, L. and M. Taylor (2002). The economics of exchange rates. Cambridge University Press. 